\title{
Molecular characterization of Echinococcus granulosus from Peru by sequencing of the mitochondrial cytochrome $C$ oxidase subunit 1 gene
}

\author{
Elizabeth Sánchez ${ }^{1,3 /+}$, Omar Cáceres ${ }^{1}$, César Náquira1, David Garcia', Gladys Patiño², \\ Herrera Silvia ${ }^{1}$, Aline C Volotão ${ }^{3}$, Octavio Fernandes ${ }^{3}$
}

${ }^{1}$ Instituto Nacional de Salud, Lima, Peru ${ }^{2}$ Hospital Hipólito Unanue, Lima, Peru ${ }^{3}$ Instituto Oswaldo Cruz-Fiocruz, Rio de Janeiro, RJ, Brasil

Echinococcus granulosus, the etiologic agent of cystic echinococcosis $(C E)$ in humans and other animal species, is distributed worldwide. Ten intra-specific variants, or genotypes (G1-G10), have been defined based on genetic diversity. To determine the genotypes present in endemic areas of Peru, samples were collected from cattle (44), sheep (41) and humans (14) from Junín, Puno Huancavelica, Cusco, Arequipa and Ayacucho. DNA was extracted from protoscolex and/or germinal layers derived from $99 \mathrm{E}$. granulosus isolates and used as templates to amplify the mitochondrial cytochrome $C$ oxidase subunit 1 gene. The resulting polymerase chain reaction products were sequenced and further examined by sequence analysis. All isolates, independent of the host, exhibited the G1 genotype. Phylogenetic analysis showed that three isolates from Ayacucho shared the same cluster with microvariant G14. The G1 genotype is considered the most widespread and infectious form of E. granulosus worldwide and our results confirm that the same patterns apply to this country. Therefore, these findings should be taken into consideration in developing prevention strategies and control programs for CE in Peru.

Key words: Echinococcus granulosus - cytochrome C oxidase subunit 1 - genotypes - Peru

Echinococcus granulosus (Batsch 1786) is a cestode, capable of infecting humans and several other animal species worldwide. The adult form resides mainly in dogs, however, the larval form (hydatid cyst) is found in a wide variety of mammals, including humans. The presence of cysts in an animal leads to cystic echinococcosis (CE). In humans, the disease is considered a critical public health problem. In addition to human health concerns, infections in cattle breeding areas result in economic losses (Thompson 2008). The disease is endemic in many South American countries, including Uruguay, Argentina, Chile, Brazil, Peru and Bolivia (Kamenetzky et al. 2002). The impact of CE on human health makes it one of the most important zoonotic diseases.

In Peru, $\mathrm{CE}$ is endemic mainly within the cattle producing areas in the central-southern regions of the country (Pérez 2007). The incidence of human cases of CE in Peru is approximately 7-11/100,000 (MS 2005), but it has been reported that in the regions of Junín, Puno, Huancavelica, Cusco, Arequipa and Ayacucho, the incidence varies between 11-39/100,000. For animal cases in these areas, the prevalence varies from $1-75 \%$ (Pérez 2007) and is primarily associated with the circulation of the parasite between sheep and dogs (Náquira 1993).

Financial support: CAPES (to ES)

+ Corresponding author: esanchez@ioc.fiocruz.br

Received 25 February 2010

Accepted 28 July 2010
As a species, E. granulosus comprises a number of intraspecific variants, strains or genotypes at the genetic level (Thompson \& McManus 2001, McManus \& Thompson 2003, Nakao et al. 2007, 2010). Currently, 10 distinct genotypes (G1-G10) have been described worldwide, based on nucleotide sequence analysis of the mitochondrial cytochrome $\mathrm{C}$ oxidase subunit $1(\mathrm{CO} 1)$ and $\mathrm{NADH}$ dehydrogenase 1 genes. These different genotypes have been associated with distinct, intermediate hosts: sheep, pigs, cattle, horses, camels, goats and cervids (Bowles et al. 1992, 1995, Bowles \& McManus 1993a, b, c, Scott et al. 1997, Zhang et al. 1998, McManus 2002, Lavikainen et al. 2003, Busi et al. 2007, Moks et al. 2008).

In South America, molecular studies have demonstrated the presence of several variants of E. granulosus. In Argentina, G1 and G6 have been isolated from human, G2 from sheep and humans and G7 from pigs (Rosenzvit et al. 1999, Kamenetzky et al. 2002). In Chile, similar studies revealed G1 and G6 in human (Manterola et al. 2008). The G1 and G6 variant have been characterized from humans in Peru (Santivañez et al. 2008). The G1 genotype has also been isolated from sheep, cattle and goats in Peru. In addition, other variants were identified, including G7 from pigs and G6 from goats (Moro et al. 2009).

It has been suggested that the extensive, intra-specific genetic variation of $E$. granulosus may be better understood within the context of variations within the life cycle pattern (Thompson \& McManus 2001). In addition, other biological consequences have been suggested to be linked to the different genotypes, such as rate of development, antigenicity, transmission profiles, sensitivity to chemotherapeutic agents and pathologic patterns (Thompson \& Lymbery 1988, Thompson et al. 1995, Thompson \& McManus 2001, 2002). 
All of these aspects should be considered in developing vaccines, diagnostic tests and pharmacological therapies for CE. Therefore, due to epidemiological implications and for the design of control strategies, it is essential that circulating E. granulosus genotypes in a given endemic area are clearly defined (McManus \& Bowles 1996, McManus \& Thompson 2003). In this study, the molecular characterization of 99 E. granulosus isolates from humans and other animals was performed by sequence analysis of a fragment of the mitochondrial CO1 gene to define the circulating genetic variants in hyper-endemic areas of Peru.

\section{MATERIALS AND METHODS}

Study area - Hydatid cysts were collected from sacrificed animals (sheep and dairy cattle) in official abattoirs from high prevalence areas of $\mathrm{CE}$ in the mountain regions of Peru (Fig. 1). The areas are known as breeding zones for dairy cattle and sheep (intermediate hosts). Moreover shepherd dogs also live in this area and are definitive hosts for parasite. In addition, human isolates were collected from hydatid cysts from patients attending different hospitals in Lima. All the samples were parasitologically confirmed at the Parasitology National Reference Laboratory [National Institute of Health (NIH) Lima, Peru].

Samples collections - All hydatid cysts were obtained under aseptic conditions. Cyst contents were aspirated and examined under light microscopy to confirm the presence of protoscolex and/or germinal layers that were washed three times with phosphate buffered saline by centrifugation at 2,000 $\mathrm{g}$ for $15 \mathrm{~min}$. The obtained pellets were kept at $95 \%$ ethanol at $-20^{\circ} \mathrm{C}$ until DNA extraction.

DNA extraction and polymerase chain reaction (PCR) - E. granulosus genomic DNA was extracted from protoscolex and/or germinal layers using the QIAmp DNA Mini Kit (QIAGEN), following the manufacturer's instructions. DNA was stored at $-20^{\circ} \mathrm{C}$ until used for PCR amplification. A region of $450 \mathrm{bp}$ of the mitochondrial CO1 gene was amplified using the following primers: 5'TTTTTTGGGCATCCTGAGGTTTAT 3' and 5' TAAAGAAAGAACATAATGAAAATG 3' (Bowles et al. 1992). The reactions were carried out in a final volume of $50 \mu \mathrm{L}$ containing approximately $50 \mathrm{ng}$ of genomic DNA in the presence of appropriate buffer with $200 \mu \mathrm{M}$ of each dNTPs, $2.5 \mathrm{mM} \mathrm{MgCl}_{2}, 50 \mathrm{pmol}$ of each primer and 1.5 units of Taq DNA polymerase (Invitrogen). The positive control was DNA from E. granulosus genotype G1 (kindly provided by Dr Mara Rosenzvit, Administración Nacional de Laboratorios e Institutos de Salud, Argentina) and negative controls were DNA isolated from Giardia duodenalis trophozoites (reference strain WB) and water. PCR reactions were performed in all samples with positive and negative controls. The thermal profile of the PCR reaction was an initial $3 \mathrm{~min}$ at $95^{\circ} \mathrm{C}$, followed by 35 cycles of 60 sec at $95^{\circ} \mathrm{C}, 60 \mathrm{sec}$ at $56^{\circ} \mathrm{C}$ and $90 \mathrm{sec}$ at $72^{\circ} \mathrm{C}$, with a final incubation at $72^{\circ} \mathrm{C}$ for $3 \mathrm{~min}$ in a Gene Amp PCR Systems 9700 (Applied Bioystems). The PCR products were analyzed with $1 \%$ agarose gel electrophoresis by ethidium bromide staining. An image of the gel was recorded under UV light using a gel documentation system (BioRad).

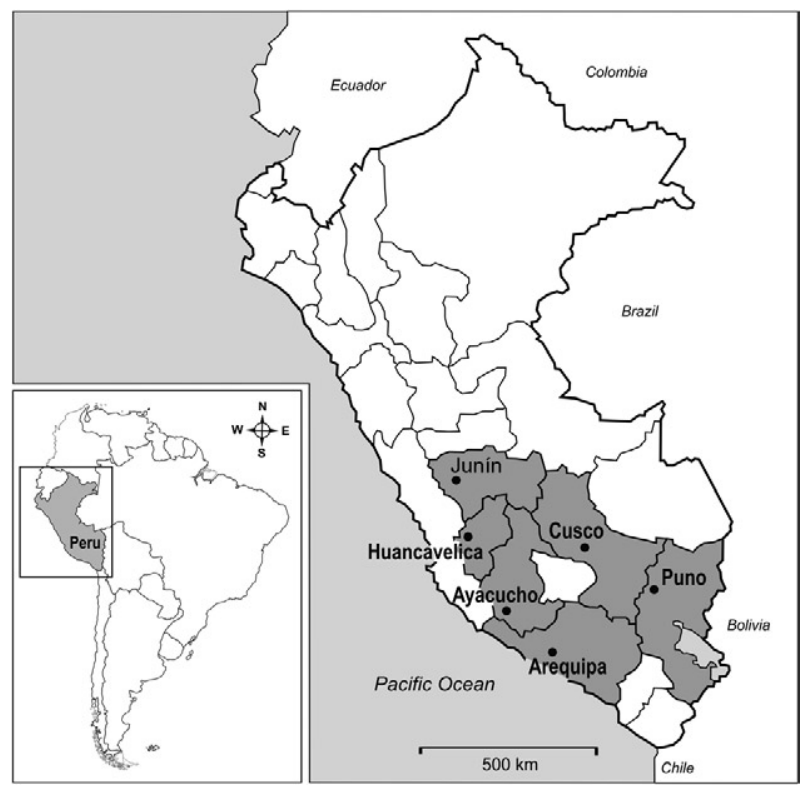

Fig. 1: geographic origins of Echinococcus granulosus isolates. A map of Peru displaying the regions in the country where samples were collected (gray regions). Inset shows location of Peru in relationships to all of South America.

Sequence analysis - PCR products were purified for sequencing using GFX PCR DNA Kit and Gel Band Purification Kit (Amersham Biosciences), following the manufacturer's instructions. The purified PCR products were sequenced using the BigDye Terminator v3.1 Cycle sequencing kit (Applied Biosystems) and analyzed by the genomic platform of DNA sequencing PDTIS/Fiocruz in an automatic sequencer ABI 3730 DNA Analyzer (Applied Biosystems), following the protocol described elsewhere (Otto et al. 2008). The chromatograms were analyzed and the nucleotide sequences obtained were aligned using the ClustalW method of the program MEGA 4.1 (megasoftware.net), using the sequences of the different genotypes of E. granulosus (G1, G2, G3, G4, G5, G6 and G7) and the microvariants of the G1 genotype (G1A, G1B, G1C, G1D, $\mathrm{G} 1 \mathrm{E}$ and $\mathrm{G} 1^{1}, \mathrm{Gl}^{2}, \mathrm{Gl}^{3}, \mathrm{G}^{4}$ ) deposited in the GenBank as references [accessions M84661/62/63/64/65/66/67 (Bowles et al. 1992), AF458871/72/73/74/75 (Kamenetzky et al. 2002) and EF393619, EF595654, EU178103, EU178104 (Vural et al. 2008)].

Phylogenetic analysis - Phylogenetic analyses were based on alignments obtained from ClustalW of a $375 \mathrm{bp}$ sequence and carried out using MEGA v4.1. The phylogenetic tree was constructed using the Neighbor-Joining algorithm (Saitou \& Nei 1987) with Kimura-2 parameters. To determine the robustness of the tree, Bootstrap analysis of 1,000 replicates was applied. A correspondent nucleotide sequence of Taenia crassiceps (GenBank accession AB03341) was used as an outgroup.

Ethic statement - This study was reviewed and approved by the Ethical Committee of NIH. 
TABLE

Geographic and host origin of Peruvian isolates of Echinococcus granulosus and the fertility of the cysts

\begin{tabular}{lccccccc}
\hline & \multicolumn{7}{c}{ E. granulosus G1 } \\
\cline { 2 - 6 } Geographic origin & Sheep & $\begin{array}{c}\text { Fertility } \\
\%\end{array}$ & Cattle & $\begin{array}{c}\text { Fertility } \\
\%\end{array}$ & Human & $\begin{array}{c}\text { Fertility } \\
\%\end{array}$ & Total \\
\hline Puno & 8 & 62.5 & 7 & 28.6 & 3 & 100 & 18 \\
Junín & 18 & 83.3 & 11 & 27.3 & 5 & 80 & 34 \\
Cusco & 0 & 0 & 9 & 44 & 1 & 100 & 10 \\
Arequipa & 2 & 100 & 12 & 0 & 1 & 100 & 15 \\
Huancavelica & 1 & 0 & 5 & 20 & 2 & 100 & 8 \\
Ayacucho $^{a}$ & 12 & 100 & 0 & 0 & 2 & 100 & 14 \\
Total & 41 & 82.9 & 44 & 22.7 & 14 & 92.85 & 99 \\
\hline
\end{tabular}

a: include $\mathrm{G} 1^{4}$ microvariants of the G1 genotype.

\section{RESULTS}

Host and geographical origins of parasites - Samples of E. granulosus cysts were obtained from six cattle producing regions of southern Peru (Fig. 1). All samples collected from sheep and cattle were obtained from animals killed in abattoirs during a one-week period from each region. Isolates were prepared in the place collected to ensure proper designation of origin. Human isolates were collected from hydatid cysts of patients attending different hospitals in Lima for the treatment of the disease. The samples were subsequently sent for parasitological confirmation at the National Reference Laboratory, NIH. Every sample was examined by light microscopy to confirm the fertility of the cysts (presence of protoscolex). The Table lists the geographical and host origins of all the isolates collected for this study, as well as the fertility of the cysts.

Amplification of CO1 and sequence analysis - The $\mathrm{CO} 1$ gene of E. granulosus has been routinely utilized for variant designation, with specific primers identified for PCR amplification (Bowles et al. 1992). In our hands, these primers generated the expected 450 bp product after PCR amplification using all 99 isolates of E. granulosus as templates. Individual PCR products were further purified after gel electrophoresis and subjected to sequencing in duplicate.

The sequences obtained were initially analyzed by multiple alignments with reported reference sequences for the G1 genotype of E. granulosus. Ninety-six analyzed sequences were demonstrated to be $100 \%$ identical to the common sheep G1 genotype (Bowles et al. 1992). Three sequences isolated from Ayacucho exhibited a single nucleotide polymorphism, a thymine residue instead of a cytosine, generating a non-synonymous change in the corresponding amino acid sequence (A/V). These sequences were identical to the $\mathrm{G} 1^{4}$ microvariant of the G1 genotype (Vural et al. 2008). Fig. 2 depicts a subset of sequences of the $\mathrm{CO} 1$ gene from the different isolates, compared to the G1 reference sequence. The obtained sequences were submitted to the GenBank database with the accession GU233854-GU233952.
Phylogenetic analysis - Phylogenetic analysis showed a robust tree clustering all samples as G1 genotype with a strong bootstrap (1,000 replicates). A particular group, composed of three sequences corresponding to $\mathrm{G} 1^{4}$ microvariants, from a single geographic region is also depicted (Fig. 3). Bootstrap values are shown at relevant nodes. The evolutionary distance between the groups is very short, suggesting that the genetic divergence is recent.

\section{DISCUSSION}

An initial step in controlling the lifecycle of E. granulosus and minimizing infections is to determine the genotype. Peru has been reported to harbor a number of different E. granulosus genotypes. The presence of G6 and G7 genotypes was confirmed by partial sequence analysis of samples for the mitochondrial CO1 and nuclear elongation factor 1 alpha genes (Moro et al. 2009). The G7 genotype was identified in pigs from Lima, a city considered to be a low endemic area of E. granulosus. The same study reported the variant G6 in goats, as well as in one human case; nevertheless, this paper reported the predominance

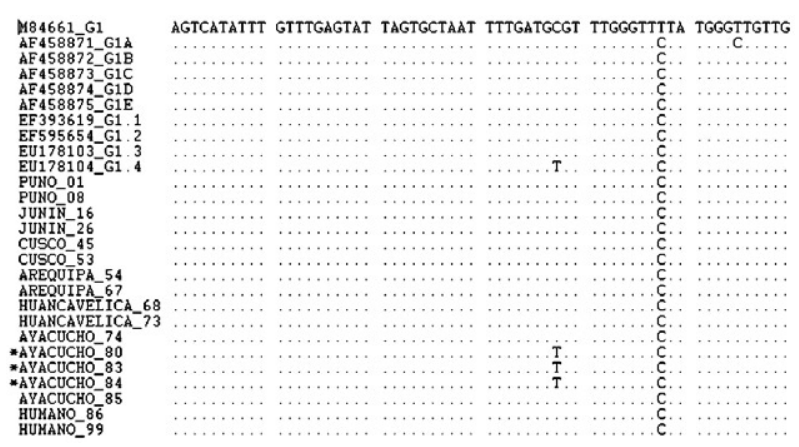

Fig. 2: sequence alignment of cytochrome $\mathrm{C}$ oxidase subunit 1 (CO1) gene. Reference sequences for the $\mathrm{CO} 1$ gene for the genotype $\mathrm{G} 1$ variant are shown at the top with a random selection of isolate sequences beneath showing identity with the G1 sequence. The three isolates with a polymorphism (denoted by asterisk) show identity with the $\mathrm{Gl}^{4}$ sequence. 


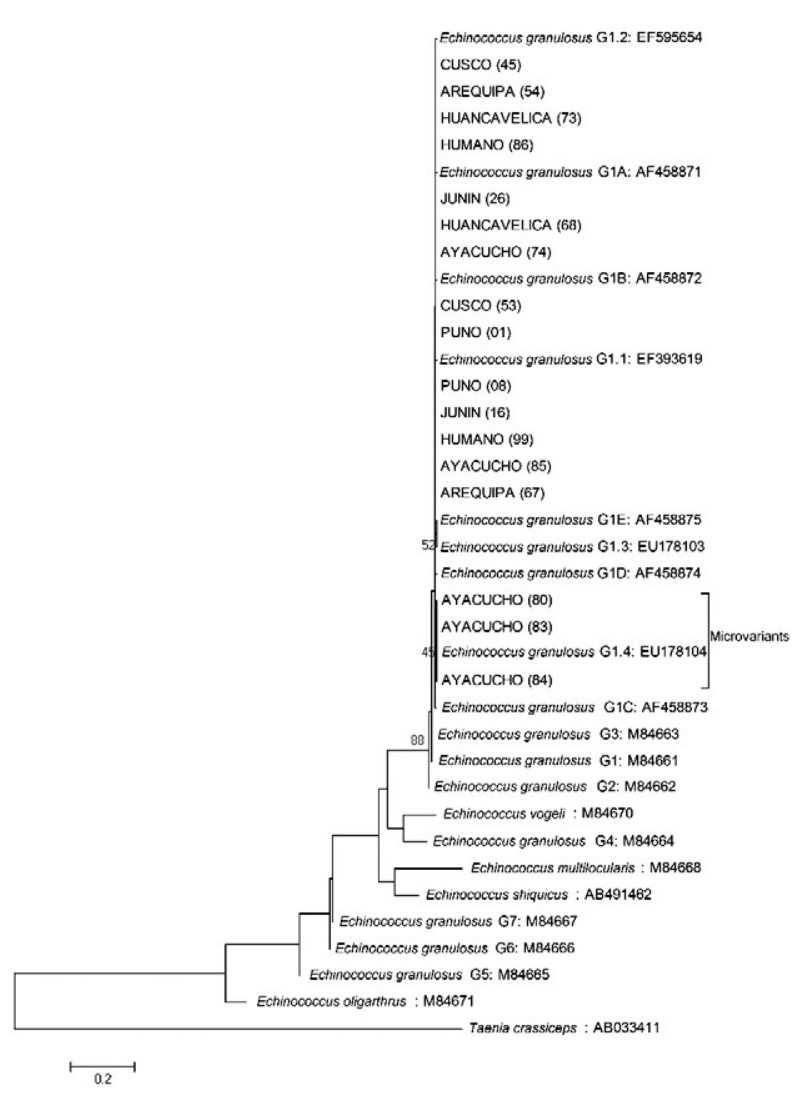

Fig. 3: phylogenetic tree of representative sequences of Echinococcus granulosus from Peru and reference sequences of other genotypes. The tree was obtained by neighbor-joining analysis using partial sequences (375 bp) from cytochrome $c$ oxidase subunit 1 gene (CO1). All isolates cluster with sequences belonging to the common sheep G1 genotype variant. The three isolates from Ayacucho with the identical one nucleotide polymorphism cluster with the $\mathrm{G} 1^{4}$ microvariants sequence (accession EU178104).

of the common sheep genotype (G1) in animal hosts and in four human cases in other studied areas of Peru. In this study, we confirmed the higher rate of fertility of G1 genotype cysts in sheep with respect to cattle.

The results presented here represent a more exhaustive sampling of cysts in cattle producing regions of Peru. Samples were obtained from cattle and sheep soon after slaughter, while human samples were collected from cysts sent to the National Reference Laboratory, NIH. Partial sequencing of the mitochondrial CO1 gene, amplified by PCR, showed the presence of only the G1 genotype among all 99 isolates studied. Similar results were reported by Santivañez et al. (2008) using the same molecular approach, showing the presence of G1 genotype in 21 human isolates from other endemic regions in Peru. G1 corresponds to the most common E. granulosus genotype found in sheep and humans worldwide.

In three of the samples from sheep in Ayachucho, a polymorphism was observed that corresponds to the $\mathrm{Gl}^{4}$ microvariant. Previously, this microvariant had been described solely in Turkey (Vural et al. 2008).
The G1 genotype is the more common, infectious $E$. granulosus genotype in the world with a wide range of hosts (Craig et al. 2003). Our discovery of a single variant suggests that similar mechanisms are responsible for its persistence in the endemic areas studied. A major consideration stems from the observation that close relationships between dogs and humans appear to correlate with the high prevalence of the disease in these areas (Moro et al. 2009). In these poorer regions, dogs are often fed with livestock viscera, which may be infected with the parasite. This activity alone could be sufficient to propagate the current endemic state. In conclusion, our results indicate the prominent circulation of the common sheep genotype (G1) in hyper-endemic areas of Peru, taking into account the substantial number of samples analyzed per area. Moreover, this paper presents the first report of $\mathrm{Gl}{ }^{4}$ microvariants of the G1 genotype in South America.

\section{ACKNOWLEDGEMENTS}

To the staff of the Regional Reference Laboratories of Puno, Junín, Cusco, Arequipa, Huancavelica and Ayacucho, Peru, for their help in samples collection, to Dr William Provance Jr, for the critical reading and English corrections of the manuscript, to Dr Mara Rosenzvit, for providing the controls genotypes, to $\mathrm{Dr}$ Adeilton Brandão, for their technical assistance, and to Heloísa Nogueira, for their help in designing the map.

\section{REFERENCES}

Bowles J, Blair D, McManus DP 1992. Genetic variants within the genus Echinococcus identified by mitochondrial DNA sequencing. Mol Biochem Parasitol 54: 165-173.

Bowles J, Blair D, McManus DP 1995. A molecular phylogeny of the genus Echinococcus. Parasitology 110: 317-328.

Bowles J, McManus DP 1993a. NADH dehydrogenase 1 gene sequences compared for species and strains of the genus Echinococcus. Int J Parasitol 23: 969-972.

Bowles J, McManus DP 1993b. Rapid discrimination of Echinococcus species and strains using a polymerase chain reaction-based RFLP method. Mol Biochem Parasitol 57: 231-239.

Bowles J, McManus DP 1993c. Molecular variation in Echinococcus. Acta Trop 53: 291-305.

Busi M, Snábel V, Varcasia A, Garippa G, Perrone V, De Liberato C, D'Amelio S 2007. Genetic variation within and between G1 and G3 genotypes of Echinococcus granulosus in Italy revealed by multilocus DNA sequencing. Vet Parasitol 150: 75-83.

Craig PS, Rogan MT, Campos-Ponce M 2003. Echinococcosis: disease, detection and transmission. Parasitology 127 (Suppl.): S5-S20.

Kamenetzky L, Gutierrez AM, Canova SG, Haag KL, Guarnera EA, Parra A, García GE, Rosenzvit MC 2002. Several strains of Echinococcus granulosus infect livestock and humans in Argentina. Infect Genet Evol 2: 129-136.

Lavikainen A, Lehtinen MJ, Meri T, Hirvelä-Koski V, Meri S 2003. Molecular genetic characterization of the Fennoscandian cervid strain, a new genotypic group (G10) of Echinococcus granulosus. Parasitology 127: 207-215.

Manterola C, Benavente F, Melo A, Vial M, Roa JC 2008. Description of Echinococcus granulosus genotypes in human hydatidosis in a region of southern Chile. Parasitol Int 57: 342-346. 
McManus DP 2002. The molecular epidemiology of Echinococcus granulosus and cystic hydatid disease. Trans $R$ Soc Trop Med Hyg 96 (Suppl. 1): S151-S157.

McManus DP, Bowles J 1996. Molecular genetic approaches to parasite identification: their value in diagnostic parasitology and systematics. Int J Parasitol 26: 687-704.

McManus DP, Thompson RC 2003. Molecular epidemiology of cystic echinococcosis. Parasitology 127 (Suppl.): S37-S51.

Moks E, Jõgisalu I, Valdmann H, Saarma U 2008. First report of Echinococcus granulosus G8 in Eurasia and a reappraisal of the phylogenetic relationships of 'genotypes' G5-G10. Parasitology 135: 647-654.

Moro PL, Nakao M, Ito A, Shantz PM, Cavero C, Cabrera L 2009. Molecular identification of Echinococcus isolates from Peru. $\mathrm{Pa}$ rasitol Int 58: 184-186.

MS - Ministério de Salud 2005. Oficina de estadística e informática, Informe Anual Estadística MINSA, MS, Lima.

Nakao M, Li T, Han X, Ma X, Xiao N, Qiu J, Wang H, Yanagida T, Mamuti W, Wen H, Moro PL, Giraudoux P, Craig PS, Ito A 2010. Genetic polymorphisms of Echinococcus tapeworms in China as determined by mitochondrial and nuclear DNA sequences. Int J Parasitol 40: 379-385.

Nakao M, McManus DP, Schantz PM, Craig PS, Ito A 2007. A molecular phylogeny of the genus Echinococcus inferred from complete mitochondrial genomes. Parasitology 134: 713-722.

Náquira C 1993. Situación de la hidatidosis en el Perú. In Memorias de la reunión del grupo científico sobre avances en la prevención, control y tratamiento de la hidatidosis, Pan American Health Organization, Washington, p. 217-229.

Otto TD, Vasconcellos EA, Gomes LH, Moreira AS, Degrave WM, Mendonça-Lima L, Alves-Ferreira M 2008. ChromaPipe: a pipeline for analysis, quality control and management for a DNA sequencing facility. Genet Mol Res 7: 861-871.

Pérez LCR 2007. Proyecto de control de hidatidosis en el Perú por vigilancia epidemiológica, PhD Thesis, Universidad Mayor de San Marcos, Lima, 132 pp.
Rosenzvit MC, Zhang LH, Kamenetzky L, Canova SG, Guarnera EA, McManus DP 1999. Genetic variation and epidemiology of Echinococcus granulosus in Argentina. Parasitology 118: 523-530.

Saitou N, Nei M 1987. The neighbor-joining method: a new method for reconstructing phylogenetic trees. Mol Biol Evol 4: 46-425.

Santivañez SJ, Gutierrez AM, Rosenzvit MC, Muzulin PM, Rodriguez ML, Vasquez JC, Rodriguez S, Gonzalez AE, Gilman RH, Garcia HH, Cysticercosis Working Group in Peru 2008. Human hydatid disease in Peru is basically restricted to Echinococcus granulosus genotypes G1. Am J Trop Med Hyg 79: 89-92.

Scott JC, Stefaniak J, Pawlowski ZS, McManus DP 1997. Molecular genetic analysis of human cystic hydatid cases from Poland: identification of a new genotypic group (G9) of Echinococcus granulosus. Parasitology 114: 37-43.

Thompson RC 2008. The taxonomy, phylogeny and transmission of Echinococcus. Exp Parasitol 119: 439-446.

Thompson RC, Lymbery AJ 1988. The nature, extent and significance of variation within the genus Echinococcus. Adv Parasitol 27: 209-258.

Thompson RC, Lymbery AJ, Constantine CC 1995. Variation in Echinococcus: towards a taxonomic revision of the genus. Adv Parasitol 35: 145-176.

Thompson RC, McManus DP 2002. Towards a taxonomic revision of the genus Echinococcus. Trends Parasitol 18: 452-457.

Thompson RCA, McManus DP 2001. Aetiology: parasites and life cyicles. In J Eckert, MA Gemmell, FX Meslin, ZS Pawlowski, Manual on Echinococcosis in humans and animals: a public health problem of global concern, World Health Organization/ World Organization for Animal Health, Paris, p. 1-9.

Vural G, Baca AU, Gauci CG, Bagci O, Gicik Y, Lightowlers MW 2008. Variability in the Echinococcus granulosus cytochrome c oxidase 1 mitochondrial gene sequence from livestock in Turkey and a reappraisal of the G1-3 genotype cluster. Vet Parasitol 154: 347-350.

Zhang L, Eslami A, Hosseini SH, McManus DP 1998. Indication of the presence of two distinct strains of Echinococcus granulosus in Iran by mitochondrial DNA markers. Am J Trop Med Hyg 59: 171-174. 